\title{
The Hawaii clopidogrel lawsuit: the possible effect on clinical laboratory testing
}

"...citizens are paying 100-times more than aspirin for an ineffective drug."

Keywords: clopidogrel $\bullet$ CYP2C19 • slow metabolizers

Rudy was a fourth-generation fisherman (name changed). His great grandfather came over from a Polynesian island a half century before Hawaii became the 50th state of the Union. Rudy worked 12-hour days, 6 days a week. After 35 years on the job, his heart told him he had to start slowing down. He suffered angina when pulling in the heavy fishing nets. His oldest son saw him grip his chest and told Rudy that he would do that part of the job from this point forward. He and his mother begged Rudy to see a cardiologist. After several months of resistance, he finally gave in.

Dr Philips was trained on the mainland and specialized in interventional cardiology. Rudy underwent an exercise stress test. Shortly after initiation on the treadmill, several of his electrographic leads showed a STsegment depression. Dr Philips immediately stopped the test. He told Rudy and his family that it was necessary to perform an elective cardiac catheterization. The procedure was scheduled for the following week. When it was concluded, Dr Philips told Rudy's family that he had significant blockage in his left anterior and circumflex arteries. Dr Philips performed angioplasty on these vessels, and inserted drug-eluting stents. Dr Philips prescribed a $75 \mathrm{mg}$ daily maintenance of clopidogrel to be taken for the next 6 months. He told Rudy that this was necessary to reduce the likelihood of postangioplasty restenosis. $\mathrm{He}$ also suggested a greatly reduced workload. Rudy was compliant with the medications. He did office work and let his son do all of the fishing. All was well until 3 months after his procedure. Rudy suffered chest pain and an ambulance was called. It appeared that he was suffering an acute myocardial infarction (AMI).

It was cases like this that prompted District Attorney (DA) David Louie of Hawaii in March of 2014 to file a lawsuit against the manufacturer of clopidogrel claiming false, unfair and deceptive marketing [1]. In a private phone call to us, Louie eluded to the basis for the suit; citizens are paying 100 times more than aspirin for an ineffective drug. Numerous studies have shown that individuals who carry at least one copy of the *2 or *3 variant have reduced hepatic 2 C19 activity converting clopidogrel to its active metabolite [2]. For these patients, the rate of acute stent thrombosis after angioplasty is significantly higher $[3,4]$.

Clopidogrel is an inhibitor of platelet aggregation and was USA FDA cleared in 1997 as an alternative to aspirin for preventing AMI and stroke. The clopidogrel versus aspirin in patients at risk of ischaemic events (CAPRIE) study of 19,185 patients compared clopidogrel versus aspirin in patients at risk for ischemic events and was used to establish the maintenance dosage of $75 \mathrm{mg} /$ day [5]. This study showed that clopidogrel reduced the relative risk by $8.7 \%$ for stroke, AMI and vascular death.

Thirteen years later, the FDA issued a black box warning stating that, 'poor metabolizers may not receive the full benefit of Plavix treatment and may remain at risk for heart attack, stroke and cardiovascular death' [6]. They suggested that doctors use alternative dosing or antiplatelet drugs for poor metabolizers. The GRAVITAS study showed

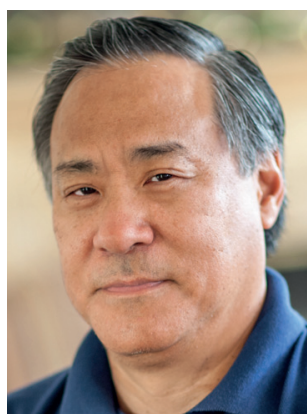

Alan HB Wu

Author for correspondence: Department of LaboratoryMedicine, University of California, San Francisco General Hospital, 1001 Potrero Ave., San Francisco, CA 94110, USA wualan@labmed2.ucsf.edu

\section{Marquitta J White} Departments of Bioengineering \& Therapeutic Sciences \& Medicine, Environments \& Health Bioengineering $\&$ Therapeutic Sciences, University of California, CA 94143, USA

\section{Sam Oh}

Departments of Bioengineering \& Therapeutic Sciences \& Medicine, Environments \& Health Bioengineering \& Therapeutic Sciences, University of California, CA 94143, USA

\section{Esteban Burchard}

Departments of Bioengineering \& Therapeutic Sciences \& Medicine, Environments \& Health Bioengineering \& Therapeutic Sciences, University of California, CA 94143, USA

and

Departments of Director, Center for Genes, Environments \& Health Bioengineering \& Therapeutic Sciences, University of California, CA 94143, USA

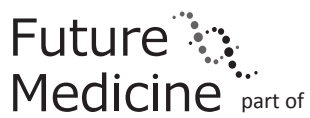


no advantage of 75 versus $150 \mathrm{mg}$ of clopidogrel for patients with a $2 \mathrm{C} 19{ }^{*} 2$ variance [7]. Others have suggested ticagrelor as an alternative antiplatelet drug for patients with CYP2C19 loss-of-function alleles [8].

In CAPRIE, 95\% of the enrolled study participants were Caucasians, where the ${ }^{*} 2$ and ${ }^{*} 3$ allele frequency is $10-20 \%$ [5]. The population of Hawaii is $1,200,000$ (42\% East Asian, 24\% Caucasians, 10\% Pacific Islander) [9]. The allele frequency for the *2 variant is 23-45\% among East Asians [10] and $40-77 \%$ among Pacific Islanders [11]. The allele frequency for the *3 variant is also higher than in Caucasians. As a total population, some 150,000-300,000 Hawaiians will have a reduced enzymatic capacity to convert clopidogrel to its active metabolite. The standard $75 \mathrm{mg}$ dose of clopidogrel is not efficacious for Hawaiians, and previous studies do not indicate that increasing the dose in the presence of the ${ }^{*} 2 /{ }^{*} 3$ alleles will increase efficacy [7].

\section{"For these patients, the rate of acute stent thromobosis after angioplasty is significantly higher."}

According to the Hawaiian Department of Health, there were 12,100 AMIs/year among Caucasians living in Hawaii and 5700 among native Hawaiians and other Pacific Islanders; translating to prevalence rates of $3.8 \%$ (Caucasians) and $4.4 \%$ (Native Hawaiians), respectively [12]. Between the years of 2007-2009, just before the FDA clearance of alternate antiplatelet drugs, the percentage of death following AMI was higher for native Hawaiians (273 total deaths/year or 4.8\%) than for Caucasians (307 deaths/year, 2.5\%) [12]. A large fraction of the patients suffering AMI in Hawaii were treated with percutaneous coronary intervention with clopidogrel given to reduce the incidence of stent thrombosis (no alternative antiplatelet medication was available). The nearly double cardiovascular death rate as a percentage of patients suffering an AMI, suggests that Hawaiians have a reduced response to clopidogrel.

Additional support for this notion comes from Oh et al. who reported the rate of premature death from heart disease broken down by US states [13]. Generally, the states with the highest mortality rates also had the highest prevalence of traditional cardiovascular disease (CVD) risk factors, in other words, hypertension, smoking, obesity and physical activity. The exception to this trend however, was the state of Hawaii; Hawaii had lower rates of these common CVD risk factors despite the high rates of premature deaths. Due to the large proportion of East Asian and Pacific Islander ancestry present in the Hawaiian population, these results may indicate additional population specific fac- tors, such as the ineffective use of clopidogrel, may be contributing to the increased CVD related mortality in comparison with the other 50 states. There are, however many other factors that affect the death rate after AMI, including co-morbidities, differences in diet, exercise and smoking rates, other genetic predispositions and access to healthcare.

Who is at fault for deaths involving failed clopidogrel? Is it the attending physician? Despite the FDA's black box warning, there are no clinical practice guidelines that have recommended 2C19 genotyping prior to clopidogrel use. Experts have argued against routine screening $[14,15,16]$. However, for certain populations that carry the alleles ${ }^{*} 2 /{ }^{*} 3$ responsible for poor metabolism, routine screening for clopidogrel metabolism and efficacy could provide an actionable step in providing effective therapeutic interventions in an 'at risk' population. Is it the clinical laboratory partly responsible for not making CYP2C19 pharmacogenomic testing available? There is no incentive for the laboratory to drive clinical testing if the demand is absent. Is the drug manufacturer at fault for unfair marketing practices? Hawaii's DA Louie claims that the manufacturers of clopidogrel have known about genetic variances and its effect on clopidogrel prior to the warning. In that case, they may be negligent in not alerting physicians, particularly those who treat minority populations where these genetic variances are prevalent.

\section{"Other antiplatelet agents such as prasugrel and ticagrelor whose efficacy is not influenced by any currently known pharmacogenomics variances is a therapeutic option."}

If the plaintiff's case is awarded, what affect will this have on CYP2C19 pharmacogenomics testing for clopidogrel? Clinical laboratories that serve a population with high prevalence for these genetic variances may consider making this test available within their clinical laboratories. Cardiology practice guidelines have recommended the use of antiplatelet agents to reduce the risk for complications after percutaneous coronary intervention [16]. If clopidogrel is determined to be the drug of choice, pharmacogenomics testing will need to be performed quickly so that a proper prescription can be scripted before the patient is discharged. Fortunately, FDA-cleared tests for CYP2C19 are commercially available. Other antiplatelet agents such as prasugrel and ticagrelor, whose efficacy is not influenced by any currently known pharmacogenomics variances is a therapeutic option for those who have CYP2C19 variances.

In our (de-identified) story, Rudy died on route to the hospital of a fatal cardiac arrhythmia. An autopsy showed a complete blockage of his circumflex artery at 
the site of his stent placement. Dr Philips was notified of Rudy's death. He wondered why the standard clopidogrel dose was insufficient in protecting his patient. Recognizing that the patient had been on clopidogrel, the forensic pathologist ordered a cytochrome P450 $2 C 19$ test using a postmortem blood sample. Indeed, Rudy had a ${ }^{*} 2 /{ }^{*} 3$ mutation. There was a memorial held for Rudy by his family on the boat where he worked. Following a eulogy, his ashes were scattered into the warm waters of the Pacific Ocean.

\section{References}

1 Orzeck K. HawaiiAG sues Plavix makers for deceptive marketing. www.law360.com

2 Gladding P, Webster M, Zeng I et al. The pharmacogentics and pharmacodynamics of clopidogrel response. JACC Cardiovasc. Interv. 1(6), 620-627 (2008).

3 Mega JL, Close SL, Wiviott SD et al. Cytochrome P-450 polymorphisms and response to clopidogrel. N. Engl. J. Med. 360(4), 354-362 (2009).

4 Shuldiner AR, O'Connell JR, Bliden KP et al. Association of cytochrome p450 2c19 genotype with antiplatelet effect and clinical efficacy of clopidogrel therapy. JAMA 302(8), 49-57 (2009).

5 CAPRIE Steering Committee. A randomized, blinded, trial of clopidogrel versus aspirin in patients at risk of ischaemic events (CAPRIE). Lancet 348, 1329-1339 (1996).

6 FDA announces new boxed warning on Plavix. Alerts patients, health care professionals to potential for reduced effectiveness. www.fda.gov

7 Price MJ, Murray SS, Angiolillo DJ et al. Influence of genetic polymorphisms on the effect of high- and standard-dose clopidogrel after percutaneous coronary intervention. J. Am. Coll. Cardiol. 59(22), 1928-1937 (2012).

8 Sorich MJ, Howowitz JD, Sorich W et al. Costeffectiveness of using CYP2C19 genotype to guide selection of clopidogrel or ticagrelor in Australia. Pharmacogenomics 14(16), 2013-2021 (2013).

9 Hawaii demographic statistics. www.infoplease.com
Financial \& competing interests disclosure

The authors have no relevant affiliations or financial involvement with any organization or entity with a financial interest in or financial conflict with the subject matter or materials discussed in the manuscript. This includes employment, consultancies, honoraria, stock ownership or options, expert testimony, grants or patents received or pending, or royalties.

No writing assistance was utilized in the production of this manuscript.

10 Sipeky C, Weber A, Szabo M et al. High prevalence of CYP2C19*2 allele in Roma samples: study of Roma and Hungarian population samples with review of the literature. Mol. Biol. Rep. 40 (8), 4727-4735 (2013).

11 Kaneko A, Lum JK, Yaviong L et al. High and variable frequencies of CYP2C19 mutations: medical consequences of poor drug metabolism in Vanuatu and other Pacific islands. Pharmacogenetics 9(5), 581-590 (1999).

12 Deaths in Hawaii due to coronary heart disease. www.hhdw.org

13 Oh SS, Croft JB, Greenlund KJ et al. Disparaties in premature deaths from heart disease- 50 States and the Disctict of Columbia. Morb. Mortal. Wkly. Rep. 53(6), 121-125 (2004).

14 Pare G, Eikelboom JW. CYP2C19 genetic testing should not be done in all patients treated with clopidogrel who are undergoing percutaneous coronary intervention. Circ. Cardiovasc. Interv. 4, 514-521 (2011).

15 Krishna V, Diamond GA, Kaul S. The role of platelet reactivity and genotype testing in the prevention of atherothrombotic cardiovascular events remains unproven. Circulation 125(10), 1288-1303 (2012).

16 Levine GN, Bates ER, Blankenship JC et al. 2011 AACC/ AHA/SCAI Guideline for percutaneous coronary intervention. Circulation 124, e574-e651 (2011). 